\title{
IMPROPER GAUSSIAN SIGNALING FOR THE TWO-USER BROADCAST CHANNEL TREATING INTERFERENCE AS NOISE
}

\author{
Christian Lameiro $^{\star}$, Ignacio Santamaría ${ }^{\dagger}$, and Peter J. Schreier ${ }^{\star}$ \\ * Signal and System Theory Group, Universität Paderborn, Germany, http://sst.upb.de \\ ${ }^{\dagger}$ Advanced Signal Processing Group, Universidad de Cantabria, Spain, http://gtas.unican.es
}

\begin{abstract}
Improper Gaussian signaling (IGS) has been shown to enlarge the rate region achievable by conventional proper Gaussian signaling (PGS) schemes in several interference-limited multiuser networks. In this work, we consider the 2-user broadcast channel (BC) when treating interference as noise "TIN" at every receiver. For this scenario, we derive a closed-form characterization of the rate region boundary with IGS. The Pareto-optimal points are achieved when at least one of the users employs maximally improper (rectilinear) signals. Differently from other interference-limited networks, our results show that IGS always outperforms PGS for the 2-user BC with TIN. Furthermore, IGS also enlarges the PGS rate region with time-sharing for this scenario.
\end{abstract}

Index Terms - Broadcast channel, improper Gaussian signaling, treating interference as noise.

\section{INTRODUCTION}

Improper Gaussian signaling (IGS) has recently been proposed as a low-complexity approach to handle interference in multiuser networks [1]. Contrary to the conventional proper Gaussian signaling (PGS), in IGS the real and imaginary parts of the transmit signals are correlated and/or have unequal power [2]. Such a statistical property has been shown to pay off in interference-limited scenarios when treating interference as noise (TIN). The benefits of IGS over PGS have been shown in, e.g., the interference channel [1,3-9], relay channels [10], and cognitive radio networks [11-13], to name a few.

The broadcast channel (BC), where a transmitter sends distinct information to a set of receivers, is one of the fundamental multiuser communication scenarios. The capacity of the BC is achieved by PGS with superposition coding and successive interference cancellation $[14,15]$. Such a nonlinear scheme, however, entails higher complexity than a linear transceiver that treats interference as noise and presents

The work of C. Lameiro and P. J. Schreier was supported by the German Research Foundation (DFG) under grants SCHR 1384/6-1 and LA 4107/1-1. The work of I. Santamaría was supported by the Ministerio de Economía y Competitividad (MINECO) and AEI/FEDER funds of the UE, Spain, under project CARMEN (TEC2016-75067-C4-4-R). also some issues regarding its practical implementation [16]. With TIN, IGS has been shown to outperform PGS in the multiantenna BC [17]. The parameters of the IGS scheme can then be optimized applying existing numerical methods. However, solutions based on numerical optimization do not permit drawing insights into the features of IGS. For example, an intriguing question is under which conditions IGS outperforms PGS. Such analytical results have been obtained, e.g., for the Z-interference channel in [9], where it was shown that IGS outperforms PGS when the ratio between the gain of the cross-link and that of the direct link is above a certain threshold. A similar result was also obtained for a cognitive radio scenario in [11].

In order to shed light onto the behavior of IGS in the $\mathrm{BC}$ with TIN, we consider a single-antenna 2-user BC. We analyze the boundary of the achievable rate region for this setting and derive closed-form expressions for the value of the parameters that attain the rate region boundary. Interestingly, our results show that all Pareto-optimal points are achieved when at least one of the users employs maximally improper signals and, unlike other interference-limited scenarios, IGS always outperforms PGS with TIN for the 2-user BC. Furthermore, there are boundary points for which one of the users transmits real-valued signals while the other uses purely imaginary signals. These boundary points strictly outperform PGS with time-sharing (TS). ${ }^{1}$ Furthermore, we show that maximally improper (rectilinear) signaling is optimal for at least one user.

\section{SYSTEM MODEL}

We consider a single-antenna 2-user BC with TIN. The received signal at the $i$ th user is given by

$$
y_{i}=h_{i}\left(\sqrt{\alpha_{1} p} s_{1}+\sqrt{\alpha_{2} p} s_{2}\right)+n_{i}, i=1,2,
$$

where $h_{i}$ is the channel coefficient of the $i$ th user, $n_{i}$ is proper Gaussian noise with variance $\sigma_{i}^{2}$, and $s_{i}$ is the signal intended to the $i$ th user, which is Gaussian with unit variance. The parameter $p$ is the transmit power, whereas $\alpha_{i}$, with $\alpha_{1}+\alpha_{2}=1$,

\footnotetext{
${ }^{1}$ We consider a TS scheme in which the power constraint is satisfied at each operating point. We refer the reader to [18] for an extended analysis of IGS with TS.
} 
determines the percentage of transmit power devoted to the $i$ th-user's message. Since the system is limited by interference, we allow the base station to transmit improper Gaussian signals. $^{2}$ Taking this into account, the achievable rate of the $i$ th user, $i=1,2$, is [4]

$R_{i}=\frac{1}{2} \log \left[\frac{\left(p g_{i}+\sigma_{i}^{2}\right)^{2}-p^{2} g_{i}^{2}\left|\alpha_{1} \kappa_{1} e^{j \phi_{1}}+\alpha_{2} \kappa_{2} e^{j \phi_{2}}\right|^{2}}{\left(\alpha_{\bar{\imath}} p g_{i}+\sigma_{i}^{2}\right)^{2}-\alpha_{\bar{\imath}}^{2} p^{2} g_{i}^{2} \kappa_{\bar{\imath}}^{2}}\right]$

where $g_{i}=\left|h_{i}\right|^{2}, \bar{\imath}=2$ if $i=1$ and $\bar{\imath}=1$ if $i=2$, and the logarithm is taken with base 2 . The parameters $\kappa_{i}$ and $\phi_{i}$ are the circularity coefficient and phase of the complementary variance, respectively, of $s_{i}$.

For this setting, we are interested in the Pareto boundary of the achievable rate region, which is the region obtained as the union of all achievable rate pairs. To this end, we first notice from (2) that both rates are simultaneously maximized by taking $\phi_{1}=\phi_{2}+\pi$. This choice can be interpreted by looking at the distribution of each user's signal. Their probability density contour are ellipses whose major axes are orthogonal to each other [2]. Therefore, since the desired signal for one user is interference for the other, this choice makes in turn the desired signal and the interference exhibit this property at both receivers simultaneously. Denoting $\alpha_{1}=\alpha$ and $\alpha_{2}=1-\alpha$, we have three parameters to optimize, namely, $\alpha, \kappa_{1}$, and $\kappa_{2}$. The achievable rates (2) can then be expressed as

$$
\begin{aligned}
& R_{1}=\frac{1}{2} \log \left[\frac{\left(p g_{1}+\sigma_{1}^{2}\right)^{2}-p^{2} g_{1}^{2}\left[\alpha \kappa_{1}-(1-\alpha) \kappa_{2}\right]^{2}}{\left[(1-\alpha) p g_{1}+\sigma_{1}^{2}\right]^{2}-(1-\alpha)^{2} p^{2} g_{1}^{2} \kappa_{2}^{2}}\right] \\
& R_{2}=\frac{1}{2} \log \left[\frac{\left(p g_{2}+\sigma_{2}^{2}\right)^{2}-p^{2} g_{2}^{2}\left[\alpha \kappa_{1}-(1-\alpha) \kappa_{2}\right]^{2}}{\left(\alpha p g_{2}+\sigma_{2}^{2}\right)^{2}-\alpha^{2} p^{2} g_{2}^{2} \kappa_{1}^{2}}\right]
\end{aligned}
$$

and the rate region is

$$
\mathcal{R}=\bigcup_{\substack{0 \leq \alpha \leq 1 \\ 0 \leq \kappa_{i} \leq 1}}\left(R_{1}, R_{2}\right)
$$

The Pareto boundary of the rate region defined above is comprised of rate pairs such that the rate of one user cannot increase without decreasing the other user's rate [19]. Therefore, each boundary point can be obtained as the solution of

$$
\begin{array}{ll}
\underset{0 \leq\left\{\alpha, \kappa_{1}, \kappa_{2}\right\} \leq 1}{\operatorname{maximize}} & R_{2}, \\
\text { subject to } & R_{1} \geq \bar{R}_{1},
\end{array}
$$

where $\bar{R}_{1}=\tau \log \left(1+\frac{p g_{1}}{\sigma_{1}^{2}}\right)$, with $0 \leq \tau \leq 1$. Varying $\tau$ between 0 and 1 and solving the above problem yields the boundary of the achievable rate region. Even though the above problem is a non-convex optimization problem, we will show in the next section that an analytical solution is possible for the global optimal point.

\footnotetext{
${ }^{2}$ See [11] for an introduction on improper random variables.
}

\section{BOUNDARY OF THE RATE REGION}

Our first result about the optimal solution of (6) is stated in the following lemma.

Lemma 1. Every point of the boundary of the rate region defined in (5) is achieved when at least one of the users employs maximally improper signaling, i.e., $\kappa_{1}=1$ and/or $\kappa_{2}=1$.

Proof. First, we can rewrite the rate constraint $R_{1} \geq \bar{R}_{1}$ as a constraint on $\kappa_{2}$, after some manipulations, as

$\kappa_{2} \geq \frac{-\alpha p g_{1} \kappa_{1}}{(1-\alpha) p g_{1} \beta_{1}}$

$+\frac{\sqrt{\beta_{1}^{2} \gamma_{1}^{2}-\beta_{1}\left(\beta_{1}+1\right) \alpha p g_{1}\left[2 \gamma_{1}-\alpha p g_{1}\right]+\left(\beta_{1}+1\right)\left(\alpha p g_{1} \kappa_{1}\right)^{2}}}{(1-\alpha) p g_{1} \beta_{1}}$,

where $\beta_{1}=2^{2 \bar{R}_{1}}-1$, and $\gamma_{1}=p g_{1}+\sigma_{1}^{2}$. We now take equality in the foregoing expression and plug it into (4), which yields an expression for $R_{2}$ that only depends on $\alpha$ and $\kappa_{1}$. Let us denote this expression as $R_{2}\left(\alpha, \kappa_{1}\right)$. Since the rate constraint on user 1 has already been accounted for with (7), the dependency of $R_{2}\left(\alpha, \kappa_{1}\right)$ on $\kappa_{1}$ can be analyzed by evaluating its derivative. By doing so, we obtain that $R_{2}\left(\alpha, \kappa_{1}\right)$ is increasing in $\kappa_{1}$ if and only if

$$
\begin{aligned}
& -\left\{2 p^{2} g_{2}^{2} \tilde{\kappa}\left[\alpha-(1-\alpha) \frac{\partial \kappa_{2}}{\partial \kappa_{1}}\right]\right\}\left[\left(\alpha p g_{2}+\sigma_{2}^{2}\right)^{2}-\left(\alpha p g_{2} \kappa_{1}\right)^{2}\right] \\
& +2\left(\alpha p g_{2}\right)^{2} \kappa_{1}\left\{\gamma_{2}^{2}-p^{2} g_{2}^{2} \tilde{\kappa}^{2}\right\} \geq 0,
\end{aligned}
$$

where $\gamma_{2}=p g_{2}+\sigma_{2}^{2}, \tilde{\kappa}=\alpha \kappa_{1}-(1-\alpha) \kappa_{2}, \kappa_{2}$ is given by (7), and

$$
\frac{\partial \kappa_{2}}{\partial \kappa_{1}}=\frac{\alpha \tilde{\kappa}}{(1-\alpha)\left[\alpha \kappa_{1}+(1-\alpha) \kappa_{2} \beta_{1}\right]} .
$$

Now we will show that condition (8) is fulfilled regardless of the values of its parameters. To this end, we plug (9) into (8), yielding

$$
\begin{aligned}
& \alpha \kappa_{1}\left[\alpha \kappa_{1}+(1-\alpha) \kappa_{2} \beta_{1}\right]\left\{\gamma_{2}^{2}-p^{2} g_{2}^{2} \tilde{\kappa}^{2}\right\} \\
& \geq(1-\alpha) \kappa_{2}\left(\beta_{1}+1\right) \tilde{\kappa}\left[\left(\alpha p g_{2}+\sigma_{2}^{2}\right)^{2}-\left(\alpha p g_{2} \kappa_{1}\right)^{2}\right] .
\end{aligned}
$$

Let us recall that $\alpha, \kappa_{1}$, and $\kappa_{2}$ belong to the interval $[0,1]$, and then $-1 \leq \tilde{\kappa} \leq 1$. The left-hand side of (10) is always positive, while the right-hand side is equal to or smaller than zero when $\bar{\kappa} \leq 0$ or, equivalently, $\alpha \kappa_{1} \leq(1-\alpha) \kappa_{2}$. Then, (10) holds for $\alpha \kappa_{1} \leq(1-\alpha) \kappa_{2}$. When this condition is not fulfilled, after some manipulations (10) can be rewritten as

$$
\begin{aligned}
& \alpha \kappa_{1} \tilde{\kappa}\left\{\gamma_{2}^{2}+p^{2} g_{2}^{2}(1-\alpha)^{2} \kappa_{2}^{2}\left(\beta_{1}+1\right)-p^{2} g_{2}^{2} \tilde{\kappa}^{2}\right\} \\
& +(1-\alpha)^{2} \kappa_{2}^{2}\left(\beta_{1}+1\right)\left(\alpha p g_{2}+\sigma_{2}^{2}\right)^{2} \\
& +\alpha \kappa_{1}(1-\alpha) \kappa_{2}\left(\beta_{1}+1\right)\left[\gamma_{2}^{2}-\left(\alpha p g_{2}+\sigma_{2}^{2}\right)^{2}\right] \geq 0 .
\end{aligned}
$$

When $\alpha \kappa_{1}>(1-\alpha) \kappa_{2}$, the first line in (11) is always nonnegative. The second line is also non-negative since all of its 
terms are non-negative. In addition, the third line is always positive because $\gamma_{2} \geq \alpha p g_{2}+\sigma_{2}^{2}$. Thus, (10) holds as well for $\alpha \kappa_{1}>(1-\alpha) \kappa_{2}$. This means that $R_{2}\left(\alpha, \kappa_{1}\right)$ is nondecreasing in $\kappa_{1}$ for all values of $\alpha$. Consequently, $R_{2}\left(\alpha, \kappa_{1}\right)$ is maximized for $\kappa_{1}=1$ except if (7) returns a value greater than one. In such a case, $\kappa_{1}$ must then be chosen as the maximum value for which $\kappa_{2}=1$. Since this is the case for any value of $\alpha$, every point of the Pareto boundary is therefore achieved by $\kappa_{1}=1$ and/or $\kappa_{2}=1$, which concludes the proof.

Lemma 1 provides an interesting result: With TIN, improper signaling outperforms proper signaling for every point of the Pareto boundary. ${ }^{3}$ Furthermore, maximally improper signaling is always optimal at least for one user. This behavior differs from other wireless scenarios, such as underlay cognitive radio networks [11], where improper signaling was shown to be optimal only when the ratio between the gains of the interference link and the direct link is above a certain threshold. In the BC channel, however, the signal and the interference for each user travel through the same channel. Furthermore, since we are considering a degraded $\mathrm{BC}$, the condition for the optimality of IGS will always be met for at least one of the users in every Pareto optimal point. Therefore, IGS with at least one user transmitting maximally improper Gaussian codewords is optimal for the 2-user BC with TIN.

In the following, we obtain the optimal transmission parameters to attain a Pareto-optimal point, namely, the values of $\kappa_{1}, \kappa_{2}$, and $\alpha$. From Lemma 1, at least one user must employ maximally improper signaling, therefore the rate region defined in (5) can be expressed as the union of two different rate regions, $\mathcal{R}=\mathcal{R}_{1} \cup \mathcal{R}_{2}$, where $\mathcal{R}_{i}$ is the achievable rate region when $\kappa_{i}=1$. The rate region $\mathcal{R}_{1}$ can be obtained by solving

$$
\begin{array}{ll}
\underset{0 \leq\left\{\alpha, \kappa_{2}\right\} \leq 1}{\operatorname{maximize}} & R_{2}, \\
\text { subject to } & R_{1} \geq \bar{R}_{1},
\end{array}
$$

with $\kappa_{1}=1$, and $\bar{R}_{1}=\frac{\tau}{2} \log \left(1+\frac{2 p g_{1}}{\sigma_{1}^{2}}\right)$. Again, varying $\tau$ between 0 and 1 we can obtain the boundary of $\mathcal{R}_{1}$. The optimal solution of (12) can be found in closed form as presented in the following lemma.

Lemma 2. The optimal solution of (12), in which $\kappa_{1}=1$, is given by

$$
\begin{aligned}
\alpha^{\star} & =\max \left(\alpha_{0}, \alpha_{t h}\right), \\
\alpha_{t h} & =\frac{\beta_{1} \sigma_{1}^{2}}{2 p g_{1}}, \alpha_{0}=\frac{-a_{1}-\sqrt{a_{1}^{2}-4 a_{2} a_{0}}}{2 a_{2}}, \\
\kappa_{2}^{\star} & =\frac{\beta_{1} \gamma_{1}-\left(\beta_{1}+2\right) \alpha^{\star} p g_{1}}{\left(1-\alpha^{\star}\right) p g_{1} \beta_{1}},
\end{aligned}
$$

\footnotetext{
${ }^{3}$ An exception is the extreme points ( $\tau=0$ or $\tau=1$ ), where only one user is active and thus proper signaling is optimal.
}

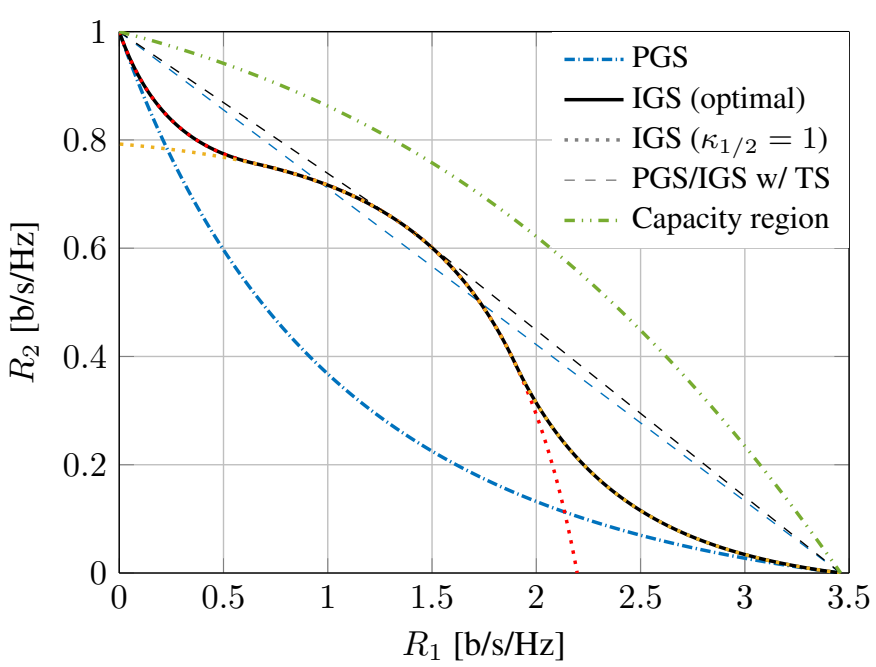

Fig. 1. Rate region boundaries for $g_{1}=10$ and $g_{2}=1$.

with

$$
\begin{aligned}
& a_{2}=-\left[\frac{2 p g_{2}\left(\beta_{1}+1\right)}{\beta_{1}}\right]^{2}, a_{1}=-4 p g_{2} \sigma_{2}^{2}\left(\frac{\beta_{1}+1}{\beta_{1}}\right)^{2}, \\
& a_{0}=\frac{g_{2}}{g_{1}} \gamma_{1}\left[\frac{g_{2}}{g_{1}} \gamma_{1}+2 \sigma_{2}^{2} \frac{\beta_{1}+1}{\beta_{1}}\right]-\gamma_{2}^{2} .
\end{aligned}
$$

Proof. For $\kappa_{1}=1$, (7) can be simplified to $\kappa_{2} \geq \kappa_{2}^{\star}$. Since $R_{2}$ is decreasing in $\kappa_{2}$, we have that $\kappa_{2}=\kappa_{2}^{\star}$ is optimal. This expression returns a valid value of $\kappa_{2}$ if it is equal to or smaller than 1, which implies $\alpha \geq \alpha_{\text {th }}$. Taking $\kappa_{2}=\kappa_{2}^{\star}$ and plugging it into (4), with $\kappa_{1}=1$, we obtain an expression for $R_{2}$, denoted as $R_{2}(\alpha)$, that depends only on $\alpha$, which yields the condition

$$
\frac{\partial R_{2}(\alpha)}{\partial \alpha} \geq 0 \Leftrightarrow \alpha^{2} a_{2}+\alpha a_{1}+a_{0} \geq 0,
$$

where $a_{2}, a_{1}$, and $a_{0}$ are given by (16), (17). First, we observe that, since $a_{2} \leq 0$, the function on the right-hand side of (18) is concave. Second, we notice that $a_{2}$ and $a_{1}$ are both equal to or smaller than zero, which, by the Descartes' rule of signs, implies that the number of positive roots of the aforementioned function is at most 1 . With these considerations, we conclude that $R_{2}(\alpha)$ is increasing in $\alpha$ for $0 \leq \alpha \leq \max \left(\alpha_{0}, 0\right)$, where $\alpha_{0}$ is such that

$$
\alpha_{0}^{2} a_{2}+\alpha_{0} a_{1}+a_{0}=0,
$$

which yields the right-hand side of (14). Finally, since $R_{2}(\alpha)$ increases monotonically in the interval $0 \leq \alpha \leq \max \left(\alpha_{0}, 0\right)$, and decreases in $\max \left(\alpha_{0}, 0\right) \leq \alpha \leq 1$, we obtain (13).

The second rate region, $\mathcal{R}_{2}$, can be obtained by solving

$$
\begin{array}{ll}
\underset{0 \leq\left\{\alpha, \kappa_{1}\right\} \leq 1}{\operatorname{maximize}} & R_{1}, \\
\text { subject to } & R_{2} \geq \bar{R}_{2},
\end{array}
$$




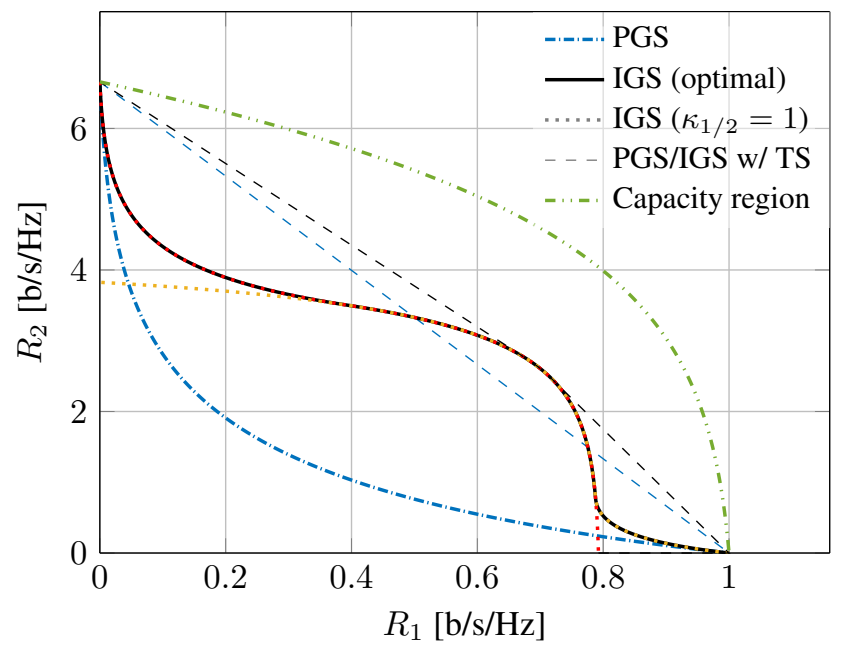

Fig. 2. Rate region boundaries for $g_{1}=1$ and $g_{2}=100$.

with $\kappa_{2}=1$, and $\bar{R}_{2}=\frac{\tau}{2} \log \left(1+\frac{2 p g_{2}}{\sigma_{2}^{2}}\right)$. Since the above problem is analogous to (12), its optimal solution is given by Lemma 2, exchanging the roles of user 1 and user 2.

\section{NUMERICAL RESULTS}

We provide some simulation examples for $p=1, \sigma_{1}^{2}=\sigma_{2}^{2}=$ 1 , and different channel gains $g_{1}, g_{2}$. Figure 1 shows the rate regions for $g_{1}=10, g_{2}=1$, and different strategies, namely: i) the capacity region achieved with superposition coding, ii) the PGS rate region, along with its convex hull (achieved with TS), and iii) the regions $\mathcal{R}_{1}$ and $\mathcal{R}_{2}$, which are obtained taking $\kappa_{1}=1$ and $\kappa_{2}=1$, respectively, along with their union and convex hull. When TS is not allowed, IGS significantly enlarges the PGS rate region. When PGS with TS is used, there is still some improvement by using IGS. Specifically, we observe that IGS enlarges the rate region for a subset of rate pairs for which both users employ maximally IGS (both users employ maximally IGS in the intersection between the rate regions corresponding to $\kappa_{1}=1$ and $\kappa_{2}=1$ ), which in turn implies an enlargement of the whole rate region if IGS with TS is employed. Indeed, the enlargement over PGS with TS is more prominent the higher the difference between both channel gains is. This observation is illustrated in Fig. 2, where $g_{1}=1$ and $g_{2}=100$. Since now user 2 is the strongest user, the enlargement of the rate region over PGS with TS is shifted to the right. Moreover, it can be observed that the enlargement is more significant than in the previous example. As a matter of fact, when both channel gains are equal, i.e., $g_{1}=g_{2}$, the capacity region becomes that of PGS with TS.

Another interesting behavior is observed from the analysis of the optimal power splitting factor $\alpha^{\star}$. Figures 3 and 4 depict this quantity for the first and second example, respectively. While for PGS the rate region boundary can be parameterized by $\alpha$, this is in general not the case for IGS.

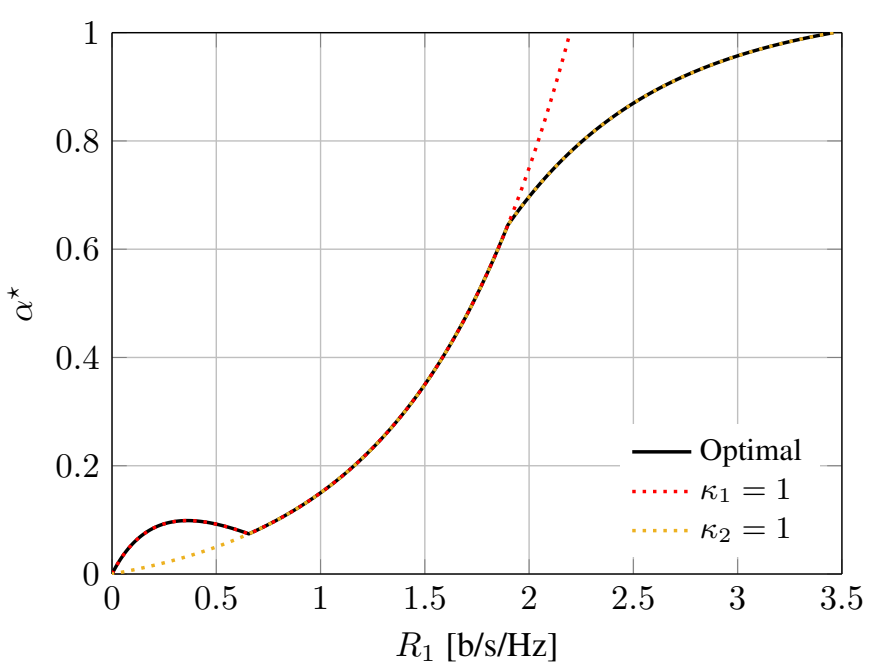

Fig. 3. Optimal power splitting for $g_{1}=10$ and $g_{2}=1$.

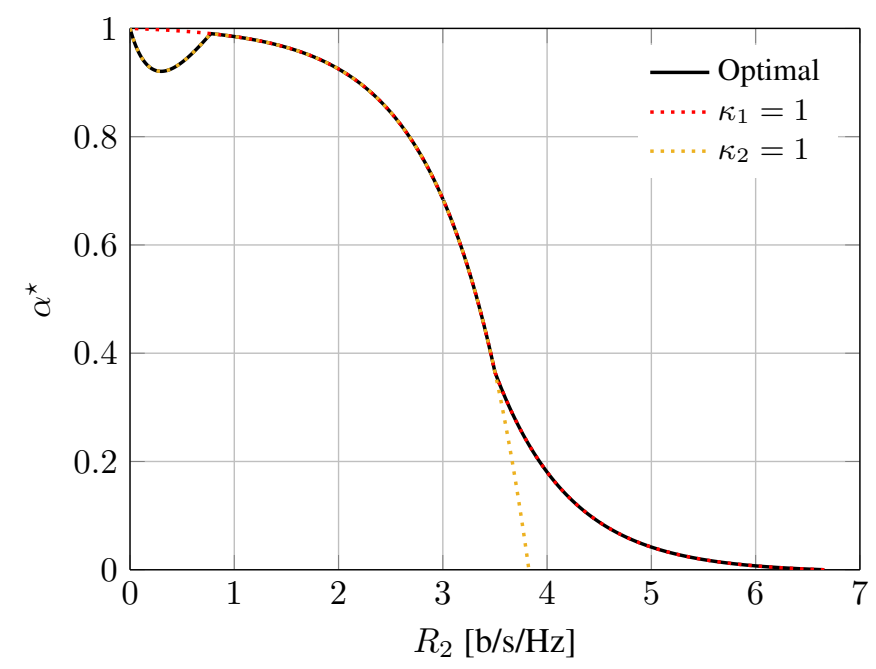

Fig. 4. Optimal power splitting for $g_{1}=1$ and $g_{2}=100$.

In particular, we observe that there are some values of $\alpha$ that correspond to up to three different points of the IGS rate region boundary. Furthermore, the protrusion observed for low values of $R_{1}\left(R_{2}\right)$ in Fig. 3 (Fig. 2) indicates that there is a subset of rate pairs for which a higher $R_{1}\left(R_{2}\right)$ is achieved by actually reducing the power allocated to this user.

\section{CONCLUSION}

We have derived a closed-form characterization of the rate region boundary for the 2-user BC with IGS+TIN. We have shown analytically that every boundary point is achieved when at least one user employs maximally IGS. Furthermore, our numerical results show that the IGS rate region is significantly larger than for PGS with and without TS. 


\section{REFERENCES}

[1] V.R. Cadambe, S.A. Jafar, and C. Wang, "Interference alignment with asymmetric complex signaling-settling the Høst-Madsen-Nosratinia conjecture," IEEE Transactions on Information Theory, vol. 56, no. 9, pp. 45524565, 2010.

[2] P.J. Schreier and L.L. Scharf, Statistical signal processing of complex-valued data: the theory of improper and noncircular signals, Cambridge, U.K.: Cambridge Univ. Press, 2010.

[3] Z.K.M. Ho and E. Jorswieck, "Improper Gaussian signaling on the two-user SISO interference channel," IEEE Transactions on Wireless Communications, vol. 11, no. 9, pp. 3194-3203, 2012.

[4] Y. Zeng, C.M. Yetis, E. Gunawan, Y.L. Guan, and R. Zhang, "Transmit optimization with improper Gaussian signaling for interference channels," IEEE Transactions on Signal Processing, vol. 61, no. 11, pp. 28992913, 2013.

[5] Y. Zeng, R. Zhang, E. Gunawan, and Y.L. Guan, "Optimized transmission with improper Gaussian signaling in the $k$-user MISO interference channel,' IEEE Transactions on Wireless Communications, vol. 12, no. 12, pp. 6303-6313, 2013.

[6] H.D. Nguyen, R. Zhang, and S. Sun, "Improper signaling for symbol error rate minimization in $K$-user interference channel," IEEE Transactions on Communications, vol. 63, no. 3, pp. 857-869, 2015.

[7] E. Kurniawan and S. Sun, "Improper Gaussian signaling scheme for the Z-interference channel," IEEE Transactions on Wireless Communications, vol. 14, no. 7, pp. 3912-3923, 2015.

[8] S. Lagen, A. Agustin, and J. Vidal, "On the superiority of improper Gaussian signaling in wireless interference MIMO scenarios," IEEE Transactions on Communications, vol. 64, no. 8, pp. 3350-3368, 2016.

[9] C. Lameiro, I. Santamaría, and P. J. Schreier, "Rate region boundary of the SISO Z-interference channel with improper signaling," IEEE Transactions on Communications, vol. 65, pp. 1022-1034, 2017.

[10] M. Gaafar, O. Amin, A. Ikhlef, A. Chaaban, and M. S. Alouini, "On alternate relaying with improper Gaussian signaling," IEEE Communications Letters, vol. 20, no. 8, pp. 1683-1686, 2016.

[11] C. Lameiro, I. Santamaría, and P.J. Schreier, "Benefits of improper signaling for underlay cognitive radio," IEEE Wireless Communications Letters, vol. 4, pp. 2225,2015 .
[12] O. Amin, W. Abediseid, and M. S. Alouini, "Underlay cognitive radio systems with improper Gaussian signaling: Outage performance analysis," IEEE Transactions on Wireless Communications, vol. 15, no. 7, pp. 48754887, 2016.

[13] M. Gaafar, O. Amin, W. Abediseid, and M. S. Alouini, "Underlay spectrum sharing techniques with in-band full-duplex systems using improper Gaussian signaling," IEEE Transactions on Wireless Communications, vol. 16, no. 1, pp. 235-249, 2017.

[14] T. Cover, "Broadcast channels," IEEE Transactions on Information Theory, vol. 18, no. 1, pp. 2-14, 1972.

[15] D. Tse and P. Viswanath, Fundamentals of Wireless Communication, Cambridge University Press, New York, NY, USA, 2005.

[16] S. Sen, N. Santhapuri, R. R. Choudhury, and S. Nelakuditi, "Successive interference cancellation: Carving out MAC layer opportunities," IEEE Transactions on Mobile Computing, vol. 12, no. 2, pp. 346-357, 2013.

[17] C. Hellings, M. Joham, and W. Utschick, "QoS feasibility in MIMO broadcast channels with widely linear transceivers," IEEE Signal Processing Letters, vol. 20, no. 11, pp. 1134-1137, 2013.

[18] C. Hellings and W. Utschick, "Improper signaling versus time-sharing in the SISO Z-interference channel," IEEE Communications Letters, vol. 21, no. 11, pp. 2432-2435, 2017.

[19] E. A. Jorswieck, E. G. Larsson, and D. Danev, "Complete characterization of the Pareto boundary for the MISO interference channel," IEEE Transactions on Signal Processing, vol. 56, no. 10, pp. 5292-5296, 2008. 